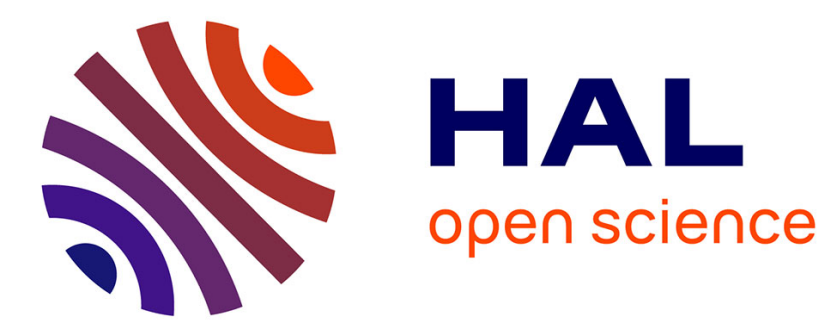

\title{
Quantum thermal bath for molecular dynamics simulation
}

Hichem Dammak, Yann Chalopin, Marine Laroche, Marc Hayoun, Jean-Jacques Greffet

\section{To cite this version:}

Hichem Dammak, Yann Chalopin, Marine Laroche, Marc Hayoun, Jean-Jacques Greffet. Quantum thermal bath for molecular dynamics simulation. Physical Review Letters, 2009, 103 (19), pp.190601. 10.1103/PhysRevLett.103.190601 . hal-00574370

\section{HAL Id: hal-00574370 https://hal-iogs.archives-ouvertes.fr/hal-00574370}

Submitted on 15 Dec 2015

HAL is a multi-disciplinary open access archive for the deposit and dissemination of scientific research documents, whether they are published or not. The documents may come from teaching and research institutions in France or abroad, or from public or private research centers.
L'archive ouverte pluridisciplinaire HAL, est destinée au dépôt et à la diffusion de documents scientifiques de niveau recherche, publiés ou non, émanant des établissements d'enseignement et de recherche français ou étrangers, des laboratoires publics ou privés. 


\title{
Quantum Thermal Bath for Molecular Dynamics Simulation
}

\author{
Hichem Dammak, ${ }^{1, *}$ Yann Chalopin, ${ }^{2}$ Marine Laroche, ${ }^{3}$ Marc Hayoun, ${ }^{4}$ and Jean-Jacques Greffet ${ }^{3}$ \\ ${ }^{1}$ Laboratoire Structures, Propriétés et Modélisation des Solides, CNRS UMR 8580, \\ Ecole Centrale Paris, F-92295 Châtenay-Malabry, France \\ ${ }^{2}$ Laboratoire d'Energétique Moléculaire et Macroscopique, CNRS UPR 288, \\ Ecole Centrale Paris, F-92295 Châtenay-Malabry, France \\ ${ }^{3}$ Laboratoire Charles Fabry, Institut d'Optique, Univ Paris Sud, CNRS, Campus Polytechnique, RD 128, 91127 Palaiseau, France \\ ${ }^{4}$ Laboratoire des Solides Irradiés, Ecole Polytechnique, CEA-DSM, CNRS, 91128 Palaiseau, France
}

(Received 15 September 2009; published 5 November 2009)

\begin{abstract}
Molecular dynamics (MD) is a numerical simulation technique based on classical mechanics. It has been taken for granted that its use is limited to a large temperature regime where classical statistics is valid. To overcome this limitation, the authors introduce in a universal way a quantum thermal bath that accounts for quantum statistics while using standard MD. The efficiency of the new technique is illustrated by reproducing several experimental data at low temperatures in a regime where quantum statistical effects cannot be neglected.
\end{abstract}

DOI: 10.1103/PhysRevLett.103.190601

Since the first work of Fermi, Pasta, and Ulam in 1955 [1], molecular dynamics (MD) simulation has frequently been used to investigate and predict the properties of condensed matter. For a crystal, these calculations are valid in the classical limit, i.e., for temperatures higher than the Debye temperature. For example, in the case of a harmonic interatomic potential, the calculated heat capacity is constant at all temperatures and equals the limit value of Dulong and Petit (1819) [2]. However, it is well known that the experimental heat capacity decreases when the temperature decreases and vanishes at $0 \mathrm{~K}$. In addition, because of the quantum fluctuations according to the Heisenberg uncertainty principle, the energy of the system at $0 \mathrm{~K}$ called "zero-point energy" is larger than the potential energy minimum. These properties are a direct consequence of the quantization of the energy of the vibration modes. These effects cannot be accounted for by using standard MD because it is based on classical statistics.

Since Planck's pioneering work, we have known that there is a profound connection between the quantization of the energy and its spectral density at thermal equilibrium. Hence, from this we get the idea of inserting in a classical treatment, random sources with a power spectral density that accounts for energy quantization. Rytov [3] has shown that introducing the power spectral density of current densities derived from the quantum fluctuation-dissipation theorem allows us to recover the blackbody radiation field. Using this approach, Lifshitz [4] has computed a pure quantum effect: the Casimir force between two metallic plates. Inspired by these works, we introduce here a general and straightforward procedure to simulate a thermal bath that allows us to include energy quantization effects into standard MD calculations.

Our approach differs from previous efforts. In the quantum molecular dynamics technique introduced by Car and
PACS numbers: 05.10.-a, 65.40.Ba, 65.40.De, 67.25.B-

Parrinello in 1985 [5], the interatomic forces are calculated quantum mechanically but the nuclei dynamics is described using standard MD. In order to include statistical quantum features into MD, several approximations have been proposed in the literature. The MD temperature can be rescaled to an effective one [6] in order to recover the mean quantum vibrational energy. However, this method does not reproduce the correct energy spectrum. The Wigner-Kirkwood approximation $[7,8]$, based on an expansion of the free energy in powers of Planck constant, can be used to correct the standard MD results but has a limited range of validity. Cao and Voth introduced the centroid molecular dynamics method [9] based on the Feynman path centroid density [10]. This technique has been widely used to study quantum correlations in liquids [11]. Very recently an approach based on a generalised Langevin equation of motion has been proposed by Wang [12] to derive the conduction heat transfer through a onedimensional linear chain of atoms between two heat baths that accounts for quantum Bose-Einstein statistics. A recent review [13] summarizes the state of the art and the need for the development of a technique that could include quantum statistical effects in MD.

In this work, we present a technique that accounts for quantum statistics by introducing a quantum thermal bath (QTB). The method is valid at any temperature and for any interatomic potential as well as for $a b$ initio schemes. The basic idea of the QTB is to use a Langevin-type approach. We introduce both a dissipative force and a Gaussian random force having the power spectral density given by the quantum fluctuation-dissipation theorem [14]. When following this approach, a difficulty arises. The power spectral density depends on the dissipation. For linear problems, the imaginary part of the response function of the system is usually well known and the power spectral 
density can be computed easily. When dealing with atomic vibrations, such information is not so well known and depends significantly on the temperature. To avoid this difficulty, we include a dissipative force that only serves the purpose of thermalizing the system. If we choose the dissipative force weak enough so that the broadening of the energy spectrum is negligible, the physical output of the model will not be affected by the dissipative force. The equation of motion of the $i$ th atom of mass $m_{i}$ obeys the Langevin-like equation

$$
m_{i} \ddot{r}_{i \alpha}=f_{i \alpha}+R_{i \alpha}-m_{i} \gamma \dot{r}_{i \alpha},
$$

where $r_{i \alpha}$ and $f_{i \alpha}$ are the $\alpha(1,2$ or 3$)$ components of the position and the force exerted by all the other atoms. The QTB is characterized by a Gaussian random force, $R_{i \alpha}$, and an effective frictional coefficient, $\gamma$. The stochastic force spectrum is not a white noise. Its power spectral density is related to $\gamma$ by the quantum mechanical fluctuationdissipation theorem [14]

$$
I_{R_{i \alpha} R_{j \beta}}(\omega)=2 m_{i} \gamma \delta_{i j} \delta_{\alpha \beta} \theta(|\omega|, T),
$$

where

$$
\theta(\omega, T)=\frac{1}{2} \hbar \omega+\hbar \omega\left[\exp \left(\hbar \omega / k_{B} T\right)-1\right]^{-1},
$$

$\delta_{i j}$ and $\delta_{\alpha \beta}$ are the Kronecker symbol and $k_{B}$ is the Boltzmann constant. It is important to emphasize that the zero-point energy contribution is taken into account through the term $\frac{1}{2} \hbar \omega$ in $\theta(\omega, T)$. The correlation function

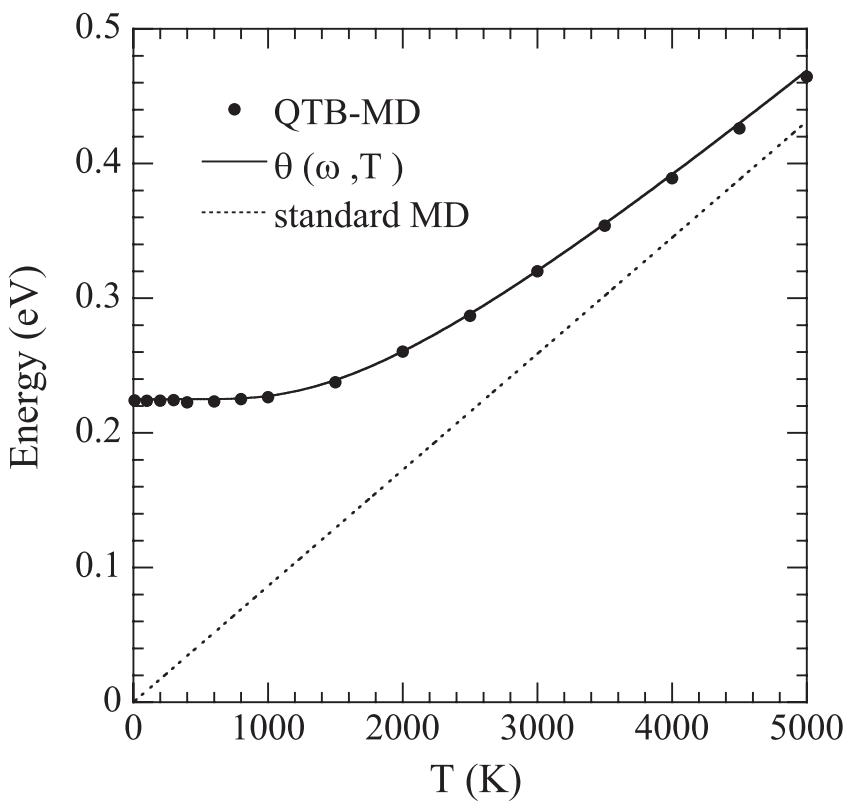

FIG. 1. QTB-MD simulation of a one-dimensional harmonic oscillator (HD molecule). The vibrational energies computed with the QTB and standard MD are compared to the expected energies given by $\theta(\omega, T) ;\left(\omega=6.84 \times 10^{14} \mathrm{rad} \mathrm{s}^{-1}\right.$ [17]). It is seen that the QTB-MD allows to recover the quantum behavior at low temperature. must satisfy the Wiener-Khinchin theorem

$$
\left\langle R_{i \alpha}(t) R_{j \beta}(t+\tau)\right\rangle=\int_{-\infty}^{+\infty} I_{R_{i \alpha} R_{j \beta}}(\omega) \exp [-i \omega \tau] \frac{d \omega}{2 \pi} .
$$

The random force $R_{i \alpha}(t)$ is computed using the numerical technique [15] designed to generate Gaussian random rough surfaces with prescribed correlation function. Note that the different random forces are uncorrelated and that the total random force does not necessarily vanish for a finite number of atoms. In order to avoid the induced collective motion of the system, $R_{i \alpha}$ is replaced by $R_{i \alpha}-$ $m_{i} \sum_{j=1}^{N} R_{j \alpha} / \sum_{j=1}^{N} m_{j}$. Finally, the coupled equations of motion are solved using standard MD algorithms [16]. The typical time to reach thermal equilibrium is about some $\gamma^{-1}$.

We first apply the method to a simple system: the HD diatomic molecule using the harmonic approximation [17]. Figure 1 shows that the QTB allows to recover the mean energy $\theta(\omega, T)$ of a quantum oscillator, whereas the standard MD predicts that the energy goes to zero at low temperature. In the case of this simple system, simulation results are independent of the effective frictional coefficient $\gamma$, provided that $\gamma \delta t \ll 1$ and the number of MD steps is large enough, where $\delta t$ is the integration time step.

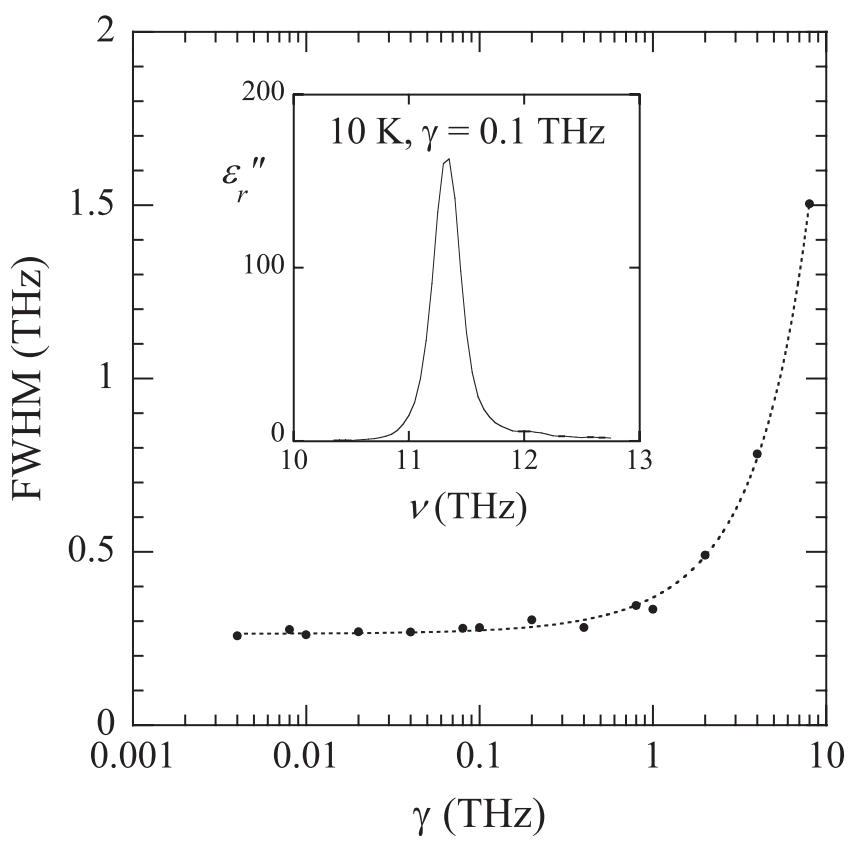

FIG. 2. Influence of the effective frictional coefficient $\gamma$. We study the infrared absorption spectrum of a $\mathrm{MgO}$ bulk at $T=$ $10 \mathrm{~K}$. Results were obtained by using a box of 64 atoms. The inset displays the imaginary part of the dielectric constant, $\varepsilon_{r}$ ", derived from the Fourier transform of the polarization correlation. The figure shows the FWHM (full width at half maximum) of the peak. 
We now apply the technique to study a $\mathrm{MgO}$ crystal using the interatomic potential proposed by Matsui [18]. We first examine whether the numerical parameter $\gamma$ influences the physical results. Figure 2 shows the influence of $\gamma$ on the simulated linewidth of the infrared absorption spectrum. It is clear that in this case, using a $\gamma$ value up to $0.3 \mathrm{THz}$ does not affect the results. More generally, $\gamma$ must be chosen lower than the linewidths of the spectral density. Figure 3 compares QTB-MD and experimental measurements [19] of the lattice parameter and the heat capacity at temperatures lower than the Debye one. The main result is that the QTB-MD allows to recover the experimental data at low temperatures whereas standard MD fails. It is important to note that the Wigner-Kirkwood quantum correction made by Matsui [18] in the case of $\mathrm{MgO}$ leads to the expected behavior of the heat capacity and the lattice parameter as a function of temperature but only above $500 \mathrm{~K}$. On the contrary, it is clear that the QTB takes into account the quantum effects at all temperatures.

Finally, we apply the technique to study ${ }^{4} \mathrm{He}$ at normal pressure and in the temperature range $2.17-4.23 \mathrm{~K}$. In these conditions, pure ${ }^{4} \mathrm{He}$ is a nonsuperfluid liquid. The crystalline state is observed for pressures higher than $\approx 25$ bar [20]. In Fig. 4, we compare the radial distribution function obtained using QTB and standard MD simulations with a Lennard-Jones (LJ) potential [21], at normal pressure and $2.5 \mathrm{~K}$. It is seen that the standard MD method leads to a stable solid state whereas the QTB-MD simulation predicts a liquid phase in agreement with experiments. The key ingredient here is that QTB-MD accounts for the zeropoint energy which is a pure quantum effect. To go beyond this qualitative result, we computed the self-diffusion coefficient from the time-dependent mean-square displacement and the Einstein formula. We found $1.9 \pm 0.1 \times$ $10^{-4} \mathrm{~cm}^{2} / \mathrm{s}$ at $4 \mathrm{~K}$ with a density of about 19.5 atom $\mathrm{nm}^{-3}$. This value is in good agreement with the experimental one estimated in the same conditions: $1.5 \pm 0.2 \times$ $10^{-4} \mathrm{~cm}^{2} / \mathrm{s}$ [22]. Using the same LJ potential and a Feynman-Hibbs approach which includes quantum corrections, a much higher value of $6.3 \times 10^{-4} \mathrm{~cm}^{2} / \mathrm{s}$ was found [26]. The path integral centroid molecular dynamics based on another empirical potential leads to a much lower value $\left(0.506 \pm 0.004 \times 10^{-4} \mathrm{~cm}^{2} / \mathrm{s}\right)$ [27]. The QTB method appears to be a simple and reliable technique to study liquids at low temperatures.

To summarize, a simple technique to generate a quantum thermal bath has been introduced. The method is easy to include in any standard MD simulation code and is independent of the system under study. It can be implemented using either phenomenological potentials or a firstprinciple description. It has been shown that thermal expansion and heat capacity of a solid can be successfully predicted at low temperatures. By accounting for the energy of quantum fluctuations, the technique allows to recover the liquid behavior of ${ }^{4} \mathrm{He}$ above the $\lambda$ point. All these examples are beyond the reach of standard MD. We think that the QTB method will significantly extend the domain of application of MD. For example, combining the
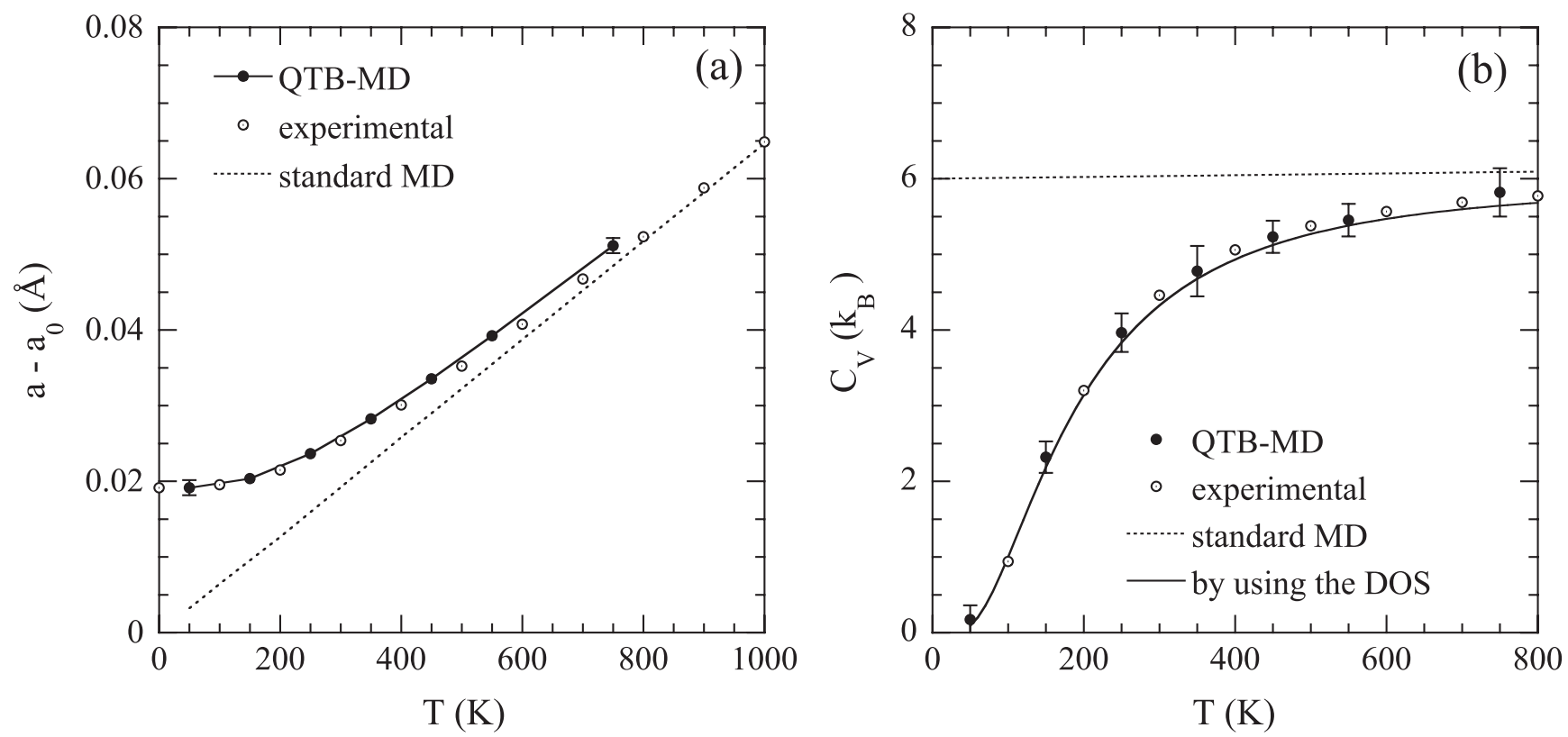

FIG. 3. QTB-MD simulation of a MgO crystal. (a) Temperature dependence of the lattice parameter, $a$. The $a_{0}$ value is obtained by extrapolating, to $0 \mathrm{~K}$, the linear behavior observed at high temperature. The QTB-MD reproduces the experimental data at low temperatures. (b) Temperature dependence of the heat capacity per molecule, $C_{V}$. The QTB values (obtained by differentiation of the mean energy) agree with the experimental data and the results derived using the harmonic density of vibrational states (DOS). The standard MD simulation gives reliable values only at temperatures higher than the Debye one (940 K) [19]. 


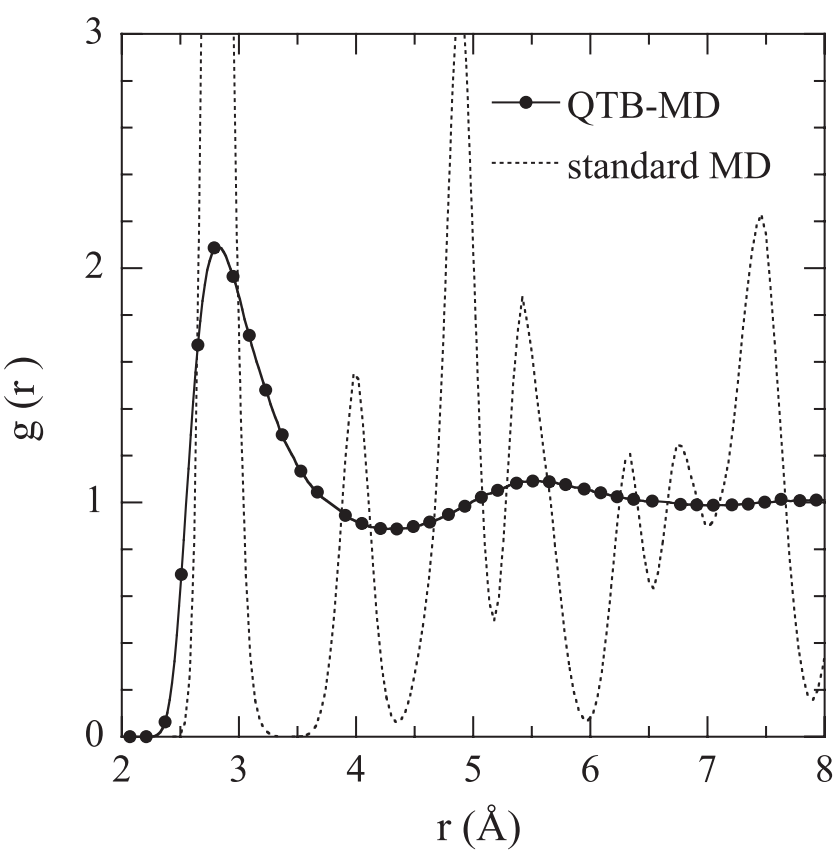

FIG. 4. QTB-MD simulation of the nonsuperfluid liquid ${ }^{4} \mathrm{He}$ at $T=2.5 \mathrm{~K}$. The feature of the radial distribution function, $g(r)$, allows to determine the phase of the simulated system: solid or liquid. $g(r)$ is calculated using both the QTB and standard MD. Results were obtained by using a box of 256 atoms. The QTBMD predicts the experimentally observed liquid phase whereas the standard MD leads to a solid one.

QTB method and the Car-Parrinello approach [5] will provide a new powerful technique including quantum effects for both electrons and nuclei.

J. J. Greffet is indebted to J. J. Sáenz with whom the initial idea of this work was generated.

*Corresponding author. hichem.dammak@ecp.fr

[1] E. Fermi, J. R. Pasta, and S. M. Ulam, Los Alamos Report No. LA-1940 1955; Collected Works of E. Fermi II (University of Chicago Press, Chicago, IL, 1965), 978.

[2] A. T. Petit and P.L. Dulong, Annals of Philosophy XIV, No. LXXIX, 189 (1819).

[3] S. M. Rytov, Sov. Phys. JETP 6, 130 (1958).
[4] E. M. Lifshitz, Zh. Eksp. Teor. Fiz. 29, 94 (1955) Sov. Phys. JETP 2, 73 (1956).

[5] R. Car and M. Parrinello, Phys. Rev. Lett. 55, 2471 (1985).

[6] C.Z. Wang, C. T. Chan, and K. M. Ho, Phys. Rev. B 42, 11276 (1990).

[7] E. Wigner, Phys. Rev. 40, 749 (1932).

[8] J. G. Kirkwood, Phys. Rev. 44, 31 (1933).

[9] J. Cao and G. A. Voth, J. Chem. Phys. 101, 6168 (1994).

[10] R.P. Feynman and A. Hibbs, Quantum Mechanics and Path-integral (McGraw-Hill, New York, 1965).

[11] U. W. Schmitt and G. A. Voth, J. Chem. Phys. 111, 9361 (1999).

[12] J. S. Wang, Phys. Rev. Lett. 99, 160601 (2007).

[13] H. M. Miller, Proc. Natl. Acad. Sci. U.S.A. 102, 6660 (2005).

[14] H. B. Callen and T. A. Welton, Phys. Rev. 83, 34 (1951).

[15] A. A. Maradudin, T. Michel, A. R. McGurn, and E. R. Mndez, Ann. Phys. (Leipzig) 203, 255 (1990).

[16] H.J.C. Berendsen and W.F. van Gunsteren, in Proceedings of the International School of Physics, "Enrico Fermi" Course 97, edited by G. Cicotti and W. G. Hoover, (North Holland Physics Publishing, Amsterdam, 1986).

[17] F. T. Prochaska and L. Andrews, J. Chem. Phys. 67, 1139 (1977).

[18] M. Matsui, J. Chem. Phys. 91, 489 (1989).

[19] O. L. Anderson and I. Suzuki, J. Geophys. Res. 88, 3549 (1983).

[20] B.L. Holian, W.D.Gwinn, and A.C. Luntz, J. Chem. Phys. 59, 5444 (1973).

[21] J. De Boer and A. Michels, Physica (Amsterdam) 5, 945 (1938).

[22] The experimental value of the self-diffusion coefficient is not available for the liquid phase but one can estimate its value from the viscosity [23] $\eta: D=\kappa \eta \rho$, where $\rho$ is the mass density and $\kappa$ a coefficient depending on the interatomic potential. From the value [24] of $\eta(3.3 \pm 0.3 \times$ $\left.10^{-6} \mathrm{Pas}\right)$ and the expected value of $\kappa(0.6)$ according to the LJ potential [25], $D$ is about $1.5 \pm 0.2 \times 10^{-4} \mathrm{~cm}^{2} / \mathrm{s}$.

[23] D. A. McQuarrie, Statistical Mechanics. (Harper's Chemistry series, New York, 1973).

[24] Experimental values available from http://webbook.nist. gov/chemistry/fluid/.

[25] R. L. Rowley and M. M. Painter, Int. J. Thermophys. 18, 1109 (1997).

[26] E. K. Goharshadi, M. Abbaspour, H. Kashani, and M. Baherololoom, Theor. Chem. Acc. 119, 355 (2008).

[27] S. Miura and S. A. Okazaki, J. Chem. Phys. 110, 4523 (1999). 